\title{
IUFOST2006/827 A molecular basis for the cancer preventive activity of tomato carotenoids
}

\author{
Y. Sharoni \\ Ben-Gurion University, Clinical Biochemistry, Faculty of Health Sciences, PO Box 653, 84105 Beer-Sheva, \\ Israel \\ yoav@bgu.ac.il
}

Carotenoids, compounds found in fruits and vegetables, have been shown to promote human health. Among the various carotenoids, lycopene has attracted the greatest interest from the medical community as well as the general public. A beneficial role for tomato lycopene has been demonstrated in diseases such as cancer, coronary heart disease, and hypertension as well as various chronic conditions. The beneficial effects have been examined extensively through epidemiological studies and biochemical investigations of the properties of lycopene and other tomato carotenoids. The epidemiological investigations have shown that high consumption of tomato products is associated with a lower risk of many types of cancers. This epidemiological-based information was translated to several intervention studies, the findings of which also support the role of tomatobased products in reduction of cancer risk. Elucidating the mechanisms responsible for cancer prevention by tomato phytonutrients is a major task in our laboratory. We recently reported that tomato-carotenoids which are well known for their antioxidant activity, act to prevent cancer also by stimulating a specific gene expression system termed 'antioxidant response element' and its major transcription factor, Nrf2. Stimulation of this system is an established mechanism for the mobilization of the body's defense system against carcinogens and other harmful compounds. By activating the antioxidant response element tomato carotenoids induce the production of phase II detoxification enzymes. These enzymes convert carcinogens, which can cause DNA mutations that lead to cancer into products that are less toxic and are readily excreted from the body. Tomato carotenoids also reduce intracellular transcriptional activity of female and male sex hormones which are strong risk factors for breast, endometrial and prostate cancer. Reducing the signal of these hormones, which is probably mediated by the Nrf2 transcription factor, effectively inhibits hormone-induced cancer cell growth. In conclusion, carotenoids can prevent cancer by two mechanisms which are both related to the activation of the Nrf2 transcription factor. The first, activated Nrf2 stimulates phase II detoxifying enzymes by binding the antioxidant response element sequences present in their promoters. The second, Nrf2 can interact directly with estrogen and androgen receptor transcriptional complexes to inhibit the induction of multiple cancer-related genes. 